\title{
Grand Challenges in Computational Materials Science: from description to prediction at all scales
}

\author{
Thomas Heine* \\ School of Engineering and Science, Jacobs University Bremen gGmbH, Bremen, Germany \\ ${ }^{*}$ Correspondence: t.heine@jacobs-university.de \\ Edited and reviewed by: \\ Alister Page, The University of Newcastle, Australia
}

Keywords: materials science, computational materials science, multiscale simulation model, density-functional theory, nanotechnology, graphene

\section{TRADITIONAL COMPUTATIONAL MATERIALS SCIENCE}

Materials science is historically linked to engineering driven by the need of materials with specific properties to manufacture infrastructure, machines, and devices. Therefore, there has always been a need for novel and better materials: stronger and lighter-weight, less expensive, easier to process, more durable, and having less environmental impact. Computational means have supported the design of tools, buildings, and vehicles. While for centuries materials have been designed in an ad hoc manner, sometimes guided by empirically developed "rules of thumb," the field changed when those rules were translated into the language of partial differential equations. The rise of computers and the finite elements method allowed first descriptions of the intrinsic materials properties, and computer simulations on the load-stress relation of buildings, of crack propagation in materials, or on the stability of composite materials became state-of-the-art with great impact on research and development in academia and industry.

\section{ADVANCED MATERIALS - MATERIALS SCIENCE BEYOND MECHANICS}

Better materials immediately enable incremental progress in engineering, for example, higher buildings or more fuel-efficient airplanes. More importantly, better materials opened the door for technological revolutions, e.g., for space technology, offshore wind farms, or high-speed trains. The field of materials science was revolutionized by the development of advanced materials that exploit properties beyond the mechanical ones: semiconductors are the foundation of computer technology; energy materials allowed batteries for mobile applications, advanced membranes, the desalination of water, etc. In the past decades, the functionality of materials moved to focus, which is closely linked with the raise of nanotechnology. We gain control over structural properties at the atomic scale, e.g., via selfassembly, while advanced microscopy and spectroscopy provide information for the detailed understanding of the properties of nanomaterials (Malkiat and Lewis, 2011). Modern applications encompass fields as diverse as chemistry (e.g., catalysis), chemical engineering (e.g., separation membranes), information storage (e.g., magnetic information storage), electrical engineering (e.g., photovoltaic cells or solidstate lighting), and many more. Numerous recent Nobel prizes for chemistry and physics have been associated with the development of new material classes with unprecedented properties, e.g., the discovery of fullerenes, of graphene, of the giant magnetoresistance, of conductive polymers, etc.

\section{MATERIALS SCIENCE - AN INTERDISCIPLINARY SCIENCE}

Materials science has always been interdisciplinary with a strong link to the fundamental sciences, chemistry, and physics. While chemistry is needed to understand the composition of natural materials and to create new ones, in physics we obtain the understanding of the materials-property relation, the precondition to suggest new applications, and to provide the basis for developing better-performing materials. Modern computational materials science is also intrinsically interdisciplinary. It was fueled by theoretical solid-state physics, allowing the atomistic and quantumtheoretical description of solids. With electronic band structure calculations the fundamental link between structure and electronic structure of materials was elucidated, and the prediction of materials with interesting electronic, magnetic, or optoelectronic properties became possible (Martin, 2008). New materials suggested by solid-state physics include (hightemperature) super conductors, topological insulators, and two-dimensional materials with extraordinary stability and electron mobility. Methods of statistical physics (Frenkel and Smit, 2001), such as Molecular Dynamics (MD) or Monte Carlo (MC) simulations and more recently, metadynamics (Martonak et al., 2003), provide the basis for the computer simulation of processes, such as diffusion, adsorption, and phase transitions. Theoretical chemistry strongly contributes to the field with its highly accurate methods to describe molecules, chemical reactions, and to predict spectroscopic data (Jensen, 2007; Helgaker et al., 2013). Massive atomistic computer simulations to study processes involving millions of atoms on the nanosecond time scale are now possible in computational biology (Freddolino et al., 2010). The same field advanced statistical methods and more recently the coarse-grain approach to bundle groups of atoms to pseudo atoms in a defined way. Biology-inspired functionality is employed in fields such as ionic transport and photovoltaics. Computer programs benefited strongly by the contributions of computational science, in particular of computational mathematics, where machine-tuned numerical algorithms have 
been developed and implemented in userfriendly libraries.

The developments with the biggest impact on computational materials science are probably density-functional theory (DFT) (Hohenberg and Kohn, 1964; Kohn and Sham, 1965) and the multiscale simulation model (Warshel and Levitt, 1976). Both methods have been recognized with the Nobel Prize in chemistry but their impact goes beyond and affects all disciplines of fundamental natural science. In principle, DFT is free of empirical parameters and can provide the electronic structure of any material (even though some intrinsic problems of DFT resulting from the difficulty in expressing the exchange-correlation potential remain to be solved). The energy and other properties can then be calculated as the expectation value of the DFT orbitals, and thus structures, electronic, and optoelectronic properties as well as spectroscopic data can be computed.

The multiscale simulation model solves a very important problem of computational materials science: finding the right balance between structural models and theoretical methods. Highly accurate methods create computational bottlenecks and allow only very small systems to be investigated, which are typically unrealistic models of the material. On the other hand, methods that can describe the entire system typically suffer from strong inaccuracies and lack predictive power. In a multiscale simulation, a local area of interest is identified and treated at the highest necessary accuracy level. This area is embedded in a larger one, which is treated with a lower-level method, and further shells can be added if necessary. Therefore, as a result of this layering, the structural model remains meaningful while the computational accuracy stays sufficient.

\section{COMPUTATIONAL MATERIALS}

\section{SCIENCE AT THE FINAL FRONTIER}

Materials science currently approaches a limit: the miniaturization of material structures reaches the atomic scale and it cannot descend beyond. This can be interpreted as final frontier of materials science. On the other hand, structural manipulation at the atomic level offers new possibilities that have not been addressed to date. In one dimension and two-dimensional materials such as graphene, this structural limit has already been reached, while the computer industry and (three-dimensional) nanotechnology are expected to hit this limit soon. The example of two-dimensional materials is intriguing: while no further miniaturization is possible as the layers cannot be made any thinner, nevertheless, new, and unprecedented properties arise. Recent investigations show that spectacular physical effects are not restricted to the Dirac electrons in graphene (Novoselov et al., 2005), but other materials such as transition metal dichalcogenides also offer properties that significantly differ from the bulk ones (Splendiani et al., 2010). In materials structured at the atomic scale, electronic quantum effects play an important role as the electronic structure is determined by the boundary conditions of the system. As we know from cluster physics, at this limit the addition, removal, or substitution of a single atom can significantly alter the properties of the material (Jena et al., 2005). Computational materials science using first-principles approaches can predict all those property changes. In fact, due to the relatively small system size, it is indeed possible to link highly accurate computer simulations with the state-ofthe-art experimental techniques to develop functional materials that employ physical effects of the electronic system, such as spin- and valleytronics.

\section{CHANGE OF PARADIGM: FROM DESCRIPTION TO PREDICTION}

Following the tradition of materials science, modern computational materials science is an interdisciplinary field that encompasses and extends the powerful portfolio of methods developed in biology, chemistry, mathematics, and physics. In return, the field applies them to the development of materials with improved properties, tailored for applications in all domains where materials are needed. Descriptive simulation has elucidated the properties of many materials. Understanding the structure-electronic structure relation and the underlying principles of the atomistic processes that occur thus, allowed the rational design of new materials with anticipated functionality. In future, computational screening, materials informatics, and combinatorial techniques, coupled with parameter-free first-principles calculations, will allow the prediction of advanced and functional materials before an attempt for synthesis is made. Two recent striking examples where computer prediction was used to guide the synthesis have been metal-organic frameworks for gas storage, where calculated gas adsorption isotherms have been the screening parameters for selecting materials to attempt synthesis (Farha et al., 2010), and the production of doped complex transition metal oxides, e.g., for $\mathrm{YBa}_{2} \mathrm{Fe}_{3} \mathrm{O}_{8}$ (Collins et al., 2014).

\section{THE BENEFIT OF THE DEVELOPMENT OF COMPUTATIONAL TECHNOLOGY}

The availability of computational resources has continuously increased over the past decades and we expect this trend to continue. The most important contributor is certainly the continuous validity of Moore's law, which has held now for 50 years since it has been phrased in 1965 , and which predicts that the number of transistors per silicon chip doubles every year. This trend is strengthened by the enormous progress made in numerical algorithms and by additional developments such as parallel computing, cloud computing, or recent GPU computing. Thus, as computational resources are continuously getting more powerful and economic, and computational methods more accurate, we can expect that the development of new materials will happen more and more in computer simulation rather than in the laboratory.

\section{THE GRAND CHALLENGES OF COMPUTATIONAL MATERIALS SCIENCE}

It is not difficult to foresee that Computational Materials Science will increasingly contribute to technological development. The link to experimental techniques via advanced spectroscopy methods has never been stronger than today. The paradigm change from descriptive to predictive science is on track, bottlenecked only by the computational power that is needed to feed multiscale models with highly accurate data from firstprinciples calculations. Simulation technology will be linked to materials informatics to allow efficient computational screening. This development is currently fueled by significant funding programs and by further progress in computer technology. 
This progress remains a challenge, but one where without doubt the combination of many incremental contributions will further advance the field.

The grand challenge of computational materials science is to go beyond the approach of structural engineering when designing new materials and to tackle the design of the electronic structure directly, open-mindedly taking advantage of collective effects such as quasi particles, selfassembly, quantum effects, and even relativity. In the exact sciences, new phenomena and effects are continuously discovered. They will provide new functionality to materials. By analyzing, understanding, and eventually exploiting these phenomena in materials, computational materials science shall serve as a creative hub between science and technology.

\section{REFERENCES}

Collins, C., Dyer, M. S., Demont, A., Chater, P. A., Thomas, M. F., Darling, G. R., et al. (2014). Computational prediction and experimental confirmation of B-site doping in $\mathrm{YBa}_{2} \mathrm{Fe}_{3} \mathrm{O}_{8}$. Chem. Sci. 5, 1493-1505.

Farha, O. K., Yazaydin, A. O., Eryazici, I., Malliakas, C. D., Hauser, B. G., Kanatzidis, M. G., et al. (2010). De novo synthesis of a metal-organic framework material featuring ultrahigh surface area and gas storage capacities. Nat. Chem. 2, 944-948.

Freddolino, P. L., Harrison, C. B., Liu, Y. X., and Schulten, K. (2010). Challenges in protein-folding simulations. Nat. Phys. 6, 751-758.

Frenkel, D., and Smit, B. (2001). Understanding Molecular Simulation. Waltham, MA: Academic Press.

Helgaker, T., Jørgensen, P., and Olsen, J. (2013). Molecular Electronic-Structure Theory. Hoboken, NJ: Wiley.

Hohenberg, P., and Kohn, W. (1964). Inhomogeneous electron gas. Phys. Rev. 136, B864-B871.

Jena, P., Khanna, S. N., and Rao, B. K. (2005). Clusters and Nano-Assemblies: Physical and Biological Systems. Singapore: World Scientific Pub Co Inc.

Jensen, F. (2007). Introduction to Computational Chemistry. Wiley.

Kohn, W., and Sham, L. J. (1965). Self-consistent equations including exchange and correlation effects. Phys. Rev. 140, A1133-A1138.

Malkiat, S. J., and Lewis, E. V. J. (2011). Understanding Nanomaterials. Boca Raton, FL: CRC Press.

Martin, R. M. (2008). Electronic Structrure: Basic Theory and Practical Methods. Cambridge: Cambridge University Press.

Martonak, R., Laio, A., and Parrinello, M. (2003). Predicting crystal structures: The Parrinello-Rahman method revisited. Phys. Rev. Lett. 90, 075503.

Novoselov, K. S., Geim, A. K., Morozov, S. V., Jiang, D., Katsnelson, M. I., Grigorieva, I. V., et al. (2005).
Two-dimensional gas of massless Dirac fermions in graphene. Nature 438, 197-200.

Splendiani, A., Sun, L., Zhang, Y. B., Li, T. S., Kim, J., Chim, C. Y., et al. (2010). Emerging photoluminescence in monolayer $\mathrm{MoS}_{2}$. Nano Lett. 10, 1271-1275.

Warshel, A., and Levitt, M. (1976). Theoretical studies of enzymic reactions - dielectric, electrostatic and steric stabilization of carbonium-ion in reaction of lysozyme. J. Mol. Biol. 103, 227-249.

Conflict of Interest Statement: The author declares that the research was conducted in the absence of any commercial or financial relationships that could be construed as a potential conflict of interest.

Received: 12 June 2014; accepted: 02 July 2014; published online: 16 July 2014.

Citation: Heine $T$ (2014) Grand Challenges in Computational Materials Science: from description to prediction at all scales. Front. Mater. 1:7. doi: 10.3389/fmats.2014.00007

This article was submitted to Computational Materials Science, a section of the journal Frontiers in Materials. Copyright (C) 2014 Heine. This is an open-access article distributed under the terms of the Creative Commons Attribution License (CC BY). The use, distribution or reproduction in other forums is permitted, provided the original author(s) or licensor are credited and that the original publication in this journal is cited, in accordance with accepted academic practice. No use, distribution or reproduction is permitted which does not comply with these terms. 